\title{
Early-Stage Growth of Mn Thin Films Electrodeposited on an Indium Tin Oxide Glass
}

\author{
M. Saitou \\ Department of Mechanical Systems Engineering, University of the Ryukyus, 1 Senbaru Nishihara-cho \\ Okinawa, 903-0213, Japan \\ E-mail: saitou@tec.u-ryukyu.ac.jp
}

doi: $10.20964 / 2018.05 .13$

Received: 9 January 2018 / Accepted: 19 February 2018 / Published: 10 April 2018

\begin{abstract}
Mn thin films grown on indium tin oxide glass in an early-stage electrodeposition were investigated using scanning electron microscopy (SEM) and X-ray diffraction (XRD). SEM images of the Mn thin films showed smooth surfaces comprising steps, terraces, and kinks. Using the SEM images of the steps, we calculated a step fluctuation defined by the standard deviation of the difference between a step and a regression line derived from the step. The step fluctuation was independent of the deposition time and decreased with the deposition temperature. This indicated that the distribution of steps remained in a steady state in an early stage of growth and $\mathrm{Mn}$ adatoms moving on the terrace, enhanced by the deposition temperature, suppressed the step fluctuation. XRD analysis revealed that the terrace surfaces were composed of only the (111) planes. The growth of the Mn thin films was consistent with the Frank-van der Merwe mode.
\end{abstract}

Keywords: Mn thin film; Early stage growth; Step; Terrace; Kink; Step fluctuation; Frank-van der Merwe mode

\section{FULL TEXT}

(C) 2018 The Authors. Published by ESG (www.electrochemsci.org). This article is an open access article distributed under the terms and conditions of the Creative Commons Attribution license (http://creativecommons.org/licenses/by/4.0/). 